\title{
The Application of Health Education and Health Promotion In Consolidation of Schistosomiasis Transmission Interrupted Period In Lake Area, China
}

\section{Kuangyu Song \\ Medical College of Nanchang University}

\section{Zifan Song}

the first clinical medical College of Nanchang University

Jun Ge

Jiangxi Provincial Institute of parastic diseases

Chunli Cao

National Institute of Parasitic Diseases

\section{Guanghan Hu}

Jiangxi Provincial Institute of parastic diseases

\section{Tiyuan Huang}

Schistosomiasis control station of Anyi county of jiangxi province

\section{Zhaojun Li}

Jiangxi provincial institute of Parastic diseases

\section{Weisheng Jiang}

Jiangxi provincial Institute of Parastic diseases

Jia Hu ( $\sim$ hujia3009@126.com )

First Affiliated Hospital of Nanchang University https://orcid.org/0000-0002-4958-7231

\section{Research article}

Keywords: Schistosomiasis, Schistosoma japonicum, Health education,Health promotion, Transmissioninterrupted areas, China

Posted Date: September 28th, 2020

DOI: https://doi.org/10.21203/rs.3.rs-75512/v1

License: (c) (i) This work is licensed under a Creative Commons Attribution 4.0 International License.

Read Full License 


\section{Abstract}

Background: In China, 387 counties had reached the criteria of schistosomiasis transmission interruption by the end of 2018, including 26 counties in Jiangxi province. In recent years, however, the intermediate host - Oncomelania hupensis and schistosomiasis cases, as well as schistosome-infected snails, reoccurred or re-emerged in 9 transmission-interrupted counties, in Jiangxi Province. Surveillance on intermediate host and disease is the main strategy of schistosomiasis control in areas where the schistosomiasis transmission interrupted in China. Mobilizing residents to discover and report snails is a good method for improving snail surveillance. However, lack of awareness and passive behavior towards schistosomiasis control of residents is the main reason for the re-occurrence and spread of snails in schistosomiasis transmission-interrupted areas in China. The objective of this study was to assess the effect of health education and health promotion model of "information communication + behavior participation + behavior encouragement" to consolidate the achievements in lake area of China, where the transmission of schistosomiasis had been interrupted. Until recent years, there are few reports on the evaluation of the effect of health education and health promotion behavior intervention. The research results of this paper will not only consolidate and develop the existing achievements of schistosomiasis control in the areas where transmission is interrupted, prevent the epidemic from rising and reviving, but also play an important role in the implementation of the national health equity including residents in schistosomiasis endemic areas in the outline of "Healthy China 2030" and the implementation of the commitments of "2030 Agenda for Sustainable Development" of the United Nations.

Methods: Two villages, Laoxia as control village and Xubo as experimental village, including its primary schools were located in Anyi County, Jiangxi Province, the typical region of lake and marshland area. The villagers aged 16-60 and pupils aged 8-10 were involved in the research. A model of interventions consisting of health education and promotion, namely, "information communication + behavior participation +behavior encouragement", was implemented in the experimental village after the baseline survey with no significant difference in knowledge, attitude, belief and practice (KABP) regarding schistosomiasis control was detected before intervention.

Results: After intervention, the percentages of villagers owning knowledge, appropriate attitude with regard to schistosomiasis control increased from $33.04 \%$ and $45.54 \%$ to $93.91 \%$, and $92.17 \%(P<0.01)$, respectively. The corresponding percentages for pupils increased from $12.88 \%$ and $6.82 \%$ to $97.64 \%$ and $62.20 \%(P<0.01)$, respectively. Both groups showed a significant increase in the percentage in believing that schistosomiasis can be eliminated, while no significant changes were observed in the two study groups of the control village. i.e., the percentages with such believing in adult villagers and pupils rose from $28.57 \%$ and $3.79 \%$ to $96.52 \%$ and $71.65 \%(P<0.01)$, respectively. In addition, both the compliance towards snail survey of adult villagers and the compliance towards knowledge dissemination of pupils were significantly increased to $81.74 \%$ and $90.55 \%$ in the experimental village $(P<0.01)$, respectively.

Conclusions: The measures of "information communication + behavior participation +behavior encouragement" can be considered as an effective model of health education and promotion for 
consolidating the achievement of schistosomiasis control in transmission-interrupted areas in China.

\section{Background}

Schistosomiasis is prevalent in 78 countries globally, with 600 million people at risk of infection in endemic areas [1]. It is listed as one of five key tropical diseases by the World Health Organization(WHO), due to its heavy diseases burden to people's health and social-economic development[2-3]. In China, schistosomiasis due to Schistosoma japonicum remains endemic in 453 counties of 12 provinces. Thanks to more than 70 years of active control efforts, there are 387 counties that reached the criteria of schistosomiasis transmission interruption by the end of 2018, including 26 counties in Jiangxi province[4]. In recent years, however, the intermediate host Oncomelania hupensis and schistosomiasis cases, as well as schistosome-infected snails, re-occurred or re-emerged in some transmission-interrupted counties, including 9 counties in Jiangxi Province[5-8].

Surveillance on snail and disease is the main strategy of schistosomiasis control in areas where the transmission of schistosomiasis had been interrupted in China. Mobilizing residents to discover and report snails is a good method for improving snail surveillance[9]. However, the lack of awareness and passive behavior towards schistosomiasis control of residents is the main reason for the re-occurrence and spread of snails in schistosomiasis transmission-interrupted areas in China[6].

Many factors with regard to the natural environment and social economy can affect the prevail of schistosomiasis, such as the distance between residential area and snail habitats, culture background, production mode, habits and customs and economy income etc.[10-11]. In order to consolidate the achievement of schistosomiasis control made in the transmission-interrupted areas, it is very important to improve the residents' compliance towards snail surveys on top of the health care and key part of surveillance by schistosomiasis professionals[12].

The role of health education and health promotion in schistosomiasis control has been highly recognized in both endemic areas and transmission-interrupted areas in China [13]. From the 1990s to the beginning of the 21st century, the epidemic situation of schistosomiasis in China was still serious. In this period, the focus of health education and health promotion was mostly in the areas where the epidemic situation was not controlled, and remarkable intervention effect had been achieved. However, until recent years, there are few literature reports on the evaluation of the effect of health education and health promotion behavior intervention in the transmission blocking area.

In order to examine effects of health education and health promotion in strengthening and consolidating the achievement of schistosomiasis control in transmission-interrupted areas, we carried out this experimental study correspondingly in Jiangxi Province.

The research results of this paper will not only consolidate and develop the existing achievements of schistosomiasis control in the areas where transmission is interrupted, prevent the epidemic from rising and reviving, but also play an important role in the implementation of the national health equity including 
residents in schistosomiasis endemic areas in the outline of "Healthy China 2030" and the implementation of the commitments of "2030 Agenda for Sustainable Development" of the United Nations.

\section{Methods}

\subsection{Study sites and target population}

The study was carried out in Anyi County, Jiangxi Province, where schistosomiasis transmission has been interrupted more than 20 years [8]. Two villages and primary schools (Laoxia and Xubu) were selected as the experimental group and the control group, respectively. Adult villagers aged 16-60 and pupils aged 810 were designated as the target population for the study.

The study villages and schools were 7 kilometers apart and similar with their natural environment, socialeconomic status, culture, people's livelihood, agricultural patterns (mainly planting rice), and school class settings. The two villages reached the criteria of schistosomiasis transmission interruption, (i.e. no intermediate host snails and schistosomiasis case were found in 5 years) in 1987. However, schistosomeinfected snails and acute schistosomiasis cases in pupils reappeared in 1997, 2004 and 2010 in these two villages.

In April 2015, snail surveys by environmental sampling method[14] were conducted by researchers within an area of $21,989 \mathrm{~m}^{2}$ of 19 environmental sites in the experimental village and an area of 19,536 $\mathrm{m}^{2}$ of 15 sites in the control village, respectively. No snail was found in both villages.

In October 2015, a total of 243 people (113 adults and 130 pupils) in the experimental village and 226 people (107 adults and 119 pupils) in the control village were subjected to stool examination by the KatoKatz technique[15] for diagnosis of S. japonicum infection. There was no case with schistosomiasis found in both villages.

\subsection{Baseline survey}

Before intervention, a baseline survey was carried out, both in the experimental village and the control village including their schools in 2015. A questionnaire about the knowledge, attitude, belief, and practice (KABP) with regard to schistosomiasis control was conducted in all study groups.

To obtain information for sample size calculations, a pre-survey was conducted in a nearby village for measuring the rates of awareness of knowledge, correct attitude, and belief-owning about schistosomiasis control in adult villagers and pupils. With $\mathrm{a}=0.05$ and a power of $80 \%$, we calculated the

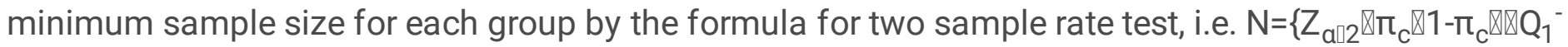
$\left.{ }^{1}+Q_{2}{ }^{-1} \square^{0.5}+Z_{\beta} \otimes \pi_{1} Q_{1}{ }^{-1} \otimes 1-\pi_{1} \otimes+\pi_{2} Q_{2}{ }^{-1} \otimes 1-\pi_{2} \square{ }^{0.5}\right\}^{2} \otimes \nabla \pi_{1}-\pi_{2} \square^{2}$. We needed at least 26 adult villagers and 25 
pupils per village. Considering a drop-out rate of $10 \%$ and respecting villagers' will to join the study, 120 adults and 140 pupils each village were enrolled for participation in the study.

Before the onset of the study, researchers had informed the study populations about the aim, content and methods of the study, and the informed consents were signed by adult villagers and pupils' legal guardian in all study groups. All people in study groups voluntarily joined this study with informed consents.

\subsubsection{Predisposing factors survey for schistosomiasis control}

A uniform questionnaire was designed and printed. The predisposing factors including knowledge, belief and attitude towards schistosomiasis were investigated in study groups.

\subsubsection{Knowledge about schistosomiasis control}

There were a total of 10 questions of knowledge about schistosomiasis, including the hazard to human health and livestock due to schistosomiasis, the presented symptoms of human advanced schistosomiasis, susceptible population and livestock, morphological identification of snail, the role of snails in schistosomiasis transmission, local snail habitats, suitable month for snail surveys, and the main measures to eliminate schistosomiasis. A total score of 60 or above (maximum possible score: 100) indicated that respondents had sufficient knowledge of schistosomiasis control. After the interview, the number of individuals who passed or did not pass and the awareness rate was recorded.

\subsubsection{Attitude towards schistosomiasis control}

The attitude of pupils to talk to their family members and others about schistosomiasis was recorded, as well as the attitude of adult villagers to participate and conduct snail surveys was recorded. The respondent who has willingness to disseminate knowledge and participate in the snail survey actively was judged as having positive attitudes towards schistosomiasis control. The number and rate of respondents with positive attitudes was also recorded.

\subsubsection{Belief of schistosomiasis control}

Asking the target population whether believe that schistosomiasis can be eliminated or not was also investigated. The respondents who believed that schistosomiasis can be eliminated were counted as the believer. The number and rate of believers were recorded.

\subsubsection{Compliance towards schistosomiasis control}

\subsubsection{Compliance towards disseminating knowledge of schistosomiasis control in pupils}

At the end of each month from March to June and September to October 2015, pupils were interviewed by investigators to understand the frequency of disseminating knowledge of schistosomiasis control 
conducted by pupils to their family members and others was recorded. The number of students who disseminated the knowledge was recorded. The compliance was defined as the percentage of students who fitted this description.

\subsubsection{Compliance towards snail survey in adult villagers}

From April to October 2015 the frequency of active snail surveys conducted by adult villagers was recorded at the end of each month, and the compliance was calculated as the percentage of adult villagers who fitted this description. A training class course on snail survey was held for the adult villagers before they conducted the survey, which consisted of exhibition of intermediate host snail samples, explaining the size, morphology of snails and snail habitats, and demonstrating and teaching how to collect snails.

\subsection{Intervention of schistosomiasis health education and health promotion}

After the baseline survey, a model of intervention of health education and promotion, namely, "information communication + behavior participation + behavior encouragement", was implemented in the experimental village from March to October, 2016.

\subsubsection{Information communication}

For pupils, a 40-minute course of health education was taught each week by school teachers and professional for schistosomiasis control, and there were a total of 4 courses during the intervention. The courses consisted of a video-tape explaining the harmful effects of schistosomiasis, explaining snail morphology, snail habitats, the role of snail in the transmission of schistosomiasis and consequences of reoccurred snails in transmission-interrupted areas with schistosomiasis, and exhibition of samples such as adult schistosomes and intermediate host. A closed book exam was held to assess the effect of teaching.

For adult villagers, information communication consisted of the following parts: (i) a similar course as for pupils was provided, including exhibits of the parasite and its intermediate host snail and a video explaining the harmful effects of the disease. (ii) health education materials regarding schistosomiasis control such as pictures, brochures, leaflets, and a bulletin board were provided to villagers. (iii) a song with regard to schistosomiasis was taught and warning signs were put up near all suspicious snail habitats to remind villagers to conduct snail surveys actively.

\subsubsection{Behavior participation}


For pupils, four kinds of activities were organized, i.e. conducting a knowledge contest on schistosomiasis control, receiving a presentation by cured schistosomiasis patients, participating in a mock snail survey on site and writing an essay about schistosomiasis control.

For adult villagers, two activities, namely, receiving a lecture by cured schistosomiasis patients and participating in a mock snail survey on site, were organized. For guaranteeing villagers to attend the lecture and join the snail survey, reverenced village heads in the study villages were mobilized to organize these activities. A souvenir, such as a towel, soap, pencil, or school bag, was given to participants after every activity was finished.

Before the mock snail survey, a training course for villagers and pupils was held to explain the snail morphology, exhibit snail samples, demonstrating the survey method and safety measures. After the survey, a reward, a towel or a pencil box, was awarded to those who found the snail samples, which were randomly bestrewed in the environments suitable for snail living by researchers on purpose.

\subsubsection{Behavior encouragement}

For pupils, the most outstanding class and individual with regard to schistosomiasis control were appraised and elected. A system of dissemination of schistosomiasis knowledge was formulated, in which each student was required to disseminate the knowledge of schistosomiasis to their family or others at least once per week. In addition, honor and reward was given to those who conducted propaganda actively.

For adult villagers, a reward system was established. i.e. honor and reward was given to those who participated in the snail survey actively, especially who can find and report snails.

\subsection{Assessment of intervention effect}

The effect of interventions was assessed both in the experimental and the control groups. The assessment content, methods and standards were used in the same way during the baseline method.

\subsection{Quality control}

\subsubsection{Personal training}

To minimize potential biases, all workers who participated in this study were well trained by researcher and they adhered to uniformed contents, methods and standards before and after the interventions.

\subsubsection{Inspection and check}


The progress and quality of this study were monitored and inspected by the principal investigator who checked the data and regularly visited the field sites to verify the authenticity of the data.

\subsection{Statistical analysis}

All data were put into a computer. A database was established with Epi Data 2.0 software, and the statistical analysis was conducted by SAS 8.2 software. The $\chi^{2}$-test was used to compare proportions before and after the implementation of health education and health promotion, with significant levels setting as 0.05 .

\section{Results}

\subsection{Knowledge about schistosomiasis control}

Before the start of the interventions, the awareness rates of knowledge in adult villagers in the experimental village and the control village were $33.04 \%$ and $32.41 \%$, respectively, with no significant difference $(P>0.05)$. For pupils in these two village, the awareness rates of knowledge were $12.88 \%$ and $10.74 \%$, respectively, also with no significant difference detected $(P>0.05)$. After intervention, the level of knowledge in the two groups in the experimental village was increased to $93.91 \%$ for adult villagers and 97.64\% for pupils, with significantly increase compared with that before intervention $(P<0.01)$, as shown in table 1. i.e $93.91 \%$ for adult villagers and $97.64 \%$ for pupils. There was no significant change in the level of knowledge in any of the two study groups in the control villages before and after intervention $(P>$ $0.05)$, i.e. $35.45 \%$ for adult villagers and $12.93 \%$ for pupils.

\section{Table 1}

General knowledge of schistosomiasis before and after health education and health promotion in two study groups, stratified by experimental and control villages, and difference between pre-and afterintervention 


\begin{tabular}{|c|c|c|c|c|c|c|}
\hline \multirow[t]{2}{*}{ Study population } & \multicolumn{2}{|c|}{ Pre-intervention } & \multicolumn{2}{|c|}{ After-intervention } & \multicolumn{2}{|c|}{ Difference } \\
\hline & \multicolumn{2}{|c|}{$\begin{array}{l}\text { No. asked No. } \\
\text { passed (\%) }\end{array}$} & \multicolumn{2}{|c|}{$\begin{array}{l}\text { No. asked No. passed } \\
(\%)\end{array}$} & \multicolumn{2}{|c|}{$\chi^{2} P$-value } \\
\hline \multicolumn{7}{|l|}{$\begin{array}{l}\text { Adult } \\
\text { residents(16- } \\
60 \text { years) }\end{array}$} \\
\hline $\begin{array}{l}\text { Experimental } \\
\text { village }\end{array}$ & 112 & $37(33.04)$ & 115 & 108(93.91) & 91.132 & 0.000 \\
\hline Control village & 108 & $35(32.41)$ & 110 & $39(35.45)$ & 0.226 & 0.635 \\
\hline \multicolumn{7}{|l|}{$\begin{array}{l}\text { Schoolchildren (8- } \\
10 \text { years) }\end{array}$} \\
\hline $\begin{array}{l}\text { Experimental } \\
\text { village }\end{array}$ & 132 & $17(12.88)$ & 127 & $124(97.64)$ & 187.477 & 0.000 \\
\hline Control village & 121 & $13(10.74)$ & 116 & $15(12.93)$ & 0.602 & 0.272 \\
\hline
\end{tabular}

\subsection{Attitude towards schistosomiasis control}

According to baseline survey, there were no significant differences in the attitude towards schistosomiasis control between the two groups of the study population in the experimental village and the control village before intervention. After intervention, the correct percentages of attitudes with regard to schistosomiasis in the experimental village were significantly higher than that before intervention with no significant difference being observed in the control village pre-and post-intervention(Table 2).

\section{Table 2}

Attitude with regard to schistosomiasis control before and after health education and health promotion in two study groups, stratified by experimental and control villages, and difference between pre-and afterintervention 


\begin{tabular}{|c|c|c|c|c|c|c|}
\hline \multirow[t]{2}{*}{ Study population } & \multicolumn{2}{|c|}{ Pre-intervention } & \multicolumn{2}{|c|}{ After-intervention } & \multicolumn{2}{|c|}{ Difference } \\
\hline & \multicolumn{2}{|c|}{$\begin{array}{l}\text { No. asked No. } \\
\text { passed }(\%)\end{array}$} & \multicolumn{2}{|c|}{$\begin{array}{l}\text { No. asked No. passed } \\
(\%)\end{array}$} & \multicolumn{2}{|c|}{$\chi^{2} P$-value } \\
\hline \multicolumn{7}{|l|}{$\begin{array}{l}\text { Adult residents }(16- \\
60 \text { years) }\end{array}$} \\
\hline Experimental village & 112 & $51(45.54)$ & 115 & 106(92.17) & 57.867 & 0.000 \\
\hline Control village & 108 & $49(45.37)$ & 110 & $56(50.91)$ & 0.670 & 0.413 \\
\hline \multicolumn{7}{|l|}{$\begin{array}{l}\text { Schoolchildren (8- } \\
10 \text { years) }\end{array}$} \\
\hline Experimental village & 132 & $9(6.82)$ & 127 & $79(62.20)$ & 88.513 & 0.000 \\
\hline Control village & 121 & 11(9.09) & 116 & $7(6.04)$ & 0.375 & 7.883 \\
\hline
\end{tabular}

\subsection{Belief of schistosomiasis control}

A similar result was obtained with regard to beliefs of schistosomiasis. Again study participants in the experimental village scored significantly better after intervention with highly significant difference, while there was no significant difference in the beliefs in the two study groups in the control villages pre-and after-intervention(Table 3).

\section{Table 3}

Belief in schistosomiasis control before and after health education and health promotion in two study groups, stratified by experimental and control villages, and difference between pre-and after-intervention

\begin{tabular}{|c|c|c|c|c|c|c|}
\hline \multirow[t]{2}{*}{ Study population } & \multicolumn{2}{|c|}{ Pre-intervention } & \multicolumn{2}{|c|}{ After-intervention } & \multicolumn{2}{|c|}{ Difference } \\
\hline & \multicolumn{2}{|c|}{$\begin{array}{l}\text { No. asked No. } \\
\text { passed (\%) }\end{array}$} & \multicolumn{2}{|c|}{$\begin{array}{l}\text { No. asked No. passed } \\
(\%)\end{array}$} & \multicolumn{2}{|c|}{$\chi^{2} P$-value } \\
\hline \multicolumn{7}{|l|}{$\begin{array}{l}\text { Adultresidents(16- } \\
60 \text { years) }\end{array}$} \\
\hline $\begin{array}{l}\text { Experimental } \\
\text { village }\end{array}$ & 112 & $32(28.57)$ & 115 & 111(96.52) & 112.385 & 0.000 \\
\hline Control village & 108 & $31(28.70)$ & 110 & $35(31.82)$ & 0.250 & 0.617 \\
\hline \multicolumn{7}{|l|}{$\begin{array}{l}\text { Schoolchildren (8- } \\
10 \text { years) }\end{array}$} \\
\hline $\begin{array}{l}\text { Experimental } \\
\text { village }\end{array}$ & 132 & $5(3.79)$ & 127 & $91(71.65)$ & 127.797 & 0.000 \\
\hline Control village & 121 & $3(2.48)$ & 116 & $6(5.17)$ & 1.176 & 0.278 \\
\hline
\end{tabular}




\subsubsection{Compliance towards snail survey in adult villagers}

Before intervention built around health education and promotion, the compliance rate with regard to participation in snail surveys in the experimental and control villages were $5.36 \% \varangle 42 / 784$ \and $6.48 \% \varangle 49 / 756)$, respectively, with no statistic difference between them $\nabla \chi$ sup $>2=0.8751, P=0.3495$. After the intervention, the corresponding figures were $81.74 \% \varangle 658 / 805 \rrbracket$ versus $7.27 \% \varangle 56 / 770 \rrbracket$ with a significant difference between them $\left(\chi^{2}=880.1160 \otimes P=0.0000\right)$. Compared with that before intervention, there was a great increase in the compliance rate towards snail survey in the experimental village after intervention, while there was no significant difference in the control village before and after intervention(Figure 1).

\subsubsection{Compliance towards dissemination of schistosomiasis knowledge in pupils}

Before intervention, there was no pupil in the two villages who talked to or educated their family members about schistosomiasis, when we surveyed 792 pupils in the experimental village and 726 pupils in the control village, respectively. After intervention, the compliance rate with regard to dissemination of schistosomiasis knowledge among pupils in the experiment village significantly increased to $90.55 \%$ $(690 / 762)\left(X^{2}=543.4797, P=0.0000\right)$. In the control village we surveyed 702 pupils there was still no pupil conducting any disseminating knowledge of schistosomiasis.

\section{Discussions}

Since the 1980s, the WHO has recommended that the health education and health promotion should be the emphasis on schistosomiasis control in order to mobilize people and actively join their efforts[16]. To date, some studies of health education and promotion have been carried out in areas where schistosomiasis is still endemic, and great effects from intervention have been achieved. In Zanzibar, influential teachers in communities were trained and intervened by employing the interactive and participatory teaching method to carry out health education about schistosomiasis for students, which make the educational services a resource for schistosomiasis control[17]. In addition, local communities co-designed schistosomiasis control interventions for school-aged children, including the implementation of novel school-based education and training, the identification of relevant safe play activities and events at local schools and the installation of community-designed urinals and laundry-washing platforms to reduce exposure schistome-infested water [18]. In another study in Zanzibar, Stothard et al. assessed the effect of a comic-strip medical booklet for the control schistosomiasis in school children but with disappointing results, which showed that heath education materials need to engage more closely with local understandings and responses to the disease if long-term sustained behavioral change is to be achieved [19]. In Uganda, it is reported that adults are increasingly rejecting free treatment of schistosomiasis and soil-transmitted helminths due to a fear of side-effects, divergence between biomedical and local understandings of schistosomiasis, as well as inappropriate and inadequate health education [20]. Considering the mass drug administration is closely related with the social, economic and 
ecological contexts, the need to adapt MDA to local circumstances is highlighted, as well as specific generalizable issues, notably with regard to health education, drug distribution and more effective use of existing public health legislation[21-22].

From the 1990s to the beginning of the 21st century, the epidemic situation of schistosomiasis in China was still serious. In this period, the focus of health education and health promotion was mostly in the areas where the epidemic situation was not controlled, and remarkable intervention effect has been achieved. In a study of health education and promotion targeting school children in heavy schistosomiasis endemic marshland areas in Jiangxi Province was carried out. Their results showed that no human schistosomiasis case was reported in eight consecutive years after intervention and schools without schistosomiasis were thriving successfully in heavy endemic areas [23]. In a 17-years study of health promotion targeting adult female residents in heavy schistosomiasis endemic areas around the Poyang Lake, the frequency of contact with snail-infested water and the infection rate declined greatly after intervention [24]. In another 18-years study of health promotion in heavy endemic areas with schistosomiasis in Poyang Lake, the compliance rate with regard to chemotherapy of adult male residents significantly increased after intervention, and new advanced schistosomiasis cases were effectively kept under control[25]. However, there is no report on the effect of health education and health promotion on maintaining the achievement made in schistosomiasis control in transmission-interrupted areas to date.

Dr. Mott pointed out in a summary that the disease control strategy formulated by WHO is practical and effective which focuses on praziquantel chemotherapy, health education and promotion and safety[26].

This paper deals with a study built around health education and promotion in schistosomiasis transmission-interrupted areas in China. Methods of psychology and praxiology were adopted in the study based on the theories of social medicine and health education. After the intervention of health education and promotion the results showed a great increase in awareness rate, correct attitude rate, the percentage of having faith with regard to schistosomiasis, the compliance rates associated with snail surveys and dissemination of schistosomiasis knowledge in the target population. These results indicate that this intervention mode is feasible and effective in further strengthening the status of transmission interruption of schistosomiasis.

Predisposing, reinforcing and enabling factors are the main three types of factors influencing human health-related behavior[27]. Predisposing factors include knowledge, attitudes, and beliefs which are the necessary condition, but not the sufficient condition for changing behavior. Enabling factors are skills or physical factors such as availability and accessibility of resources, or services that facilitate achievement of motivation to change behavior. Reinforcing factors include factors that reward or reinforce the desired behavior change, including social support, economic rewards, and changing social norms. Only these three factors work together so that the unhealthy behavior can be changed [28-29]. In this study, the intervention model of "information communication + behavior participation + behavior encouragement" was correspondent with the three factors mentioned above, respectively. First, information 
communication is the predisposing factor, which can improve the level of knowledge, attitude and beliefs towards schistosomiasis control in study groups. Second, behavior participation is the measure of enabling factors, such as four activities of school children with regard to schistosomiasis control, education conducted by old schistosomiasis patients to adult villagers and participating in a simulated snail survey in the field ${ }^{[30]}$. These enabling factors can improve the compliance of school children towards dissemination of schistosomiasis knowledge to their family members and other people as well as the compliance of adult villages to participate schistosomiasis control activities including snail surveys. Third, behavior encouragement is an enforcing factor to strengthen the study group's compliance towards participating in schistosomiasis control[31]. For the school children, the compliance towards schistosomiasis propaganda was enforced by launching a reward system in recognition of outstanding class and individual[32]. For adult villagers, the compliance towards snail survey also can be improved by rewarding anyone who can find a snail or by influence of propaganda conducted by school children and others.

The overall results discussed here demonstrate the strong potential of health education and promotion in not only raising the level of knowledge and awareness regarding to schistosomiasis control but also improving the compliance to participate in schistosomiasis control all study groups. Thus, health education and promotion carried out in schistosomiasis transmission-interrupted areas has the merit for sustaining the achievement of schistosomiasis control and eliminating schistosomiasis thoroughly.

In addition, schistosomiasis health education should be carried out by the schistosomiasis control institutions in the transmission interruption area for the local people who go out to other epidemic areas and return to other epidemic areas, other epidemic areas and local people, as well as the livestock owners who introduce livestock from the epidemic areas, so as to improve their awareness of schistosomiasis control and strictly prevent imported infectious sources.

\section{Conclusions}

The present study aims to examine effects of health education and health promotion in strengthening and consolidating the achievement of schistosomiasis control in transmission-interrupted areas. A model "information communication + behavior participation + behavior encouragement" of interventions consisting of health education and promotion was implemented in the experimental village.The percentages of villagers owning knowledge, appropriate attitude with regard to schistosomiasis control was significantly improved .Both groups showed a significant increase in the percentage in believing that schistosomiasis can be eliminated.

\section{Declarations}

The study is supported by the Ministry of Science and Technology of the People's Republic of China (Grant No.2009BAI78B00). The authors have no other relevant affiliations or financial involvementwith 
any organization or entity with a financial interest in or financial conflict with the subject matter or materials discussed in the manuscript apart fromthose disclosed.

\section{Ethics approval and consent to participate}

All data were obtained from publicly available data sources, supplied and analysed in an anonymous format, without access to personal identifying information. Therefore, our study was exempt from the ethical committee of Jiangxi institute of parasitic diseases, China. All authors declare that they approve this version of the manuscript.

\section{Consent for publication}

Not applicable.

\section{Availability of data and materials}

The data that support the findings of this study are available from the corresponding author upon reasonable request.

\section{Competing interests}

The authors declare that they have no competing interests.

\section{Funding}

This work was supported by the Ministry of Science and Technology of the People's Republic of China (Grant No.2009BAI78B00 ). The funders play no role in the study.

\section{Authors' contributions}

$\mathrm{JH}, \mathrm{KYS}$ and GHH conceived and designed the project. JH, KYS and JG wrote the manuscript, FZS, CLC and $\mathrm{JH}$ revised the manuscript. JG, GHH, TYH, ZJL, and WSJ implemented field work. All other authors contributed to editing and all authors have read and approved the final paper.

\section{Author details}

${ }^{1}$ School of Basic Medical Sciences, Nanchang University, Nanchang, Jiangxi , China. ${ }^{2}$ The First Clinical Medical College of Nanchang University , Nanchang, Jiangxi , China. ${ }^{3}$ Jiangxi Provincial Institute of Parasitic Diseases, Nanchang, Jiangxi ,China.

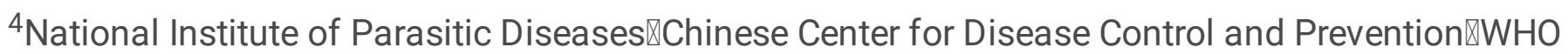

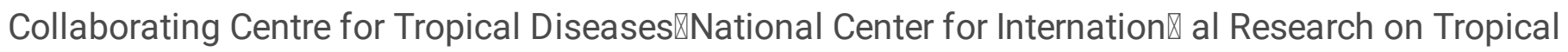

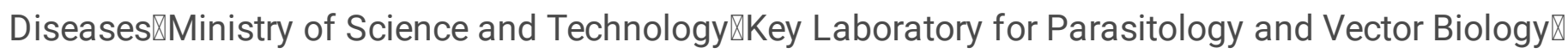

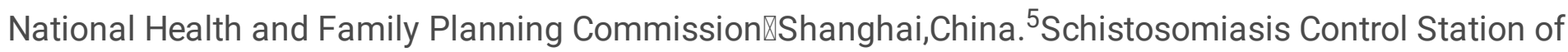


Anyi County, Anyi ,Jiangxi , China. ${ }^{6}$ The First Affiliated Hospital of Nanchang University , Nanchang , Jiangxi ,China.

\section{Acknowledgements}

Not applicablehis

\section{References}

1. WHO,2013. Schistosomiasis: progress report 2002-2011,strategic plan 2012-2020.World Health Organization,Geneva.

2. WHO. Report of the Scientific Working Group meeting on Schistosomiasis. Geneva: World Health Organization; 2005.

3. Chen M-G. Use of praziquantel for clinical treatment and morbidity control of schistosomiasis japonica in China: a review of 30 years' experience. Acta Trop. 2005;96:168-76.

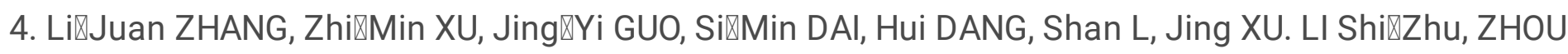

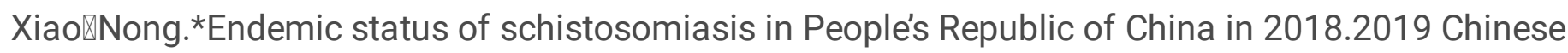
Journal of Schistosomiasis Control, 6,576-582.

5. LIU. Yi®Wen,GE Jun,LIN Dan®Dan.Challenges and countermeasures of schistosomiasis elimination in Jiangxi Province. 2018. Chin J Schisto Control,6.610-614.

6. Gen-Quan QI, Guang-Han HU, Ren-Sheng Y, Chen-Zi YOU. Zhang Ju,Ge Jun.2007. KAP of residents on schistosomiasis control in transmission-interrupted areas. Chin. J. Schisto. Contr. 1,53-55.

7. LIU. Xiao®Zhong,CHEN Zhe,LIN Dan囚dan.Status and future development of surveillance of schistosomiasis in Jiangxi Province.2016.Chin J Schisto Control 2.111-140.

8. Ti-Yuan HUANG, Guang-han HU,JIANG Xun-Fang GAO, Jian-Ping GE, Jun XIE, Xing-Fu,XING Yong. Endemic situation of schistosomiasis after transmission-interupted in Anyi County: a 23-year surveillance.2010.Chin J Schisto Control, 6,624,633.

9. Yang Kun, Wang Wei, LÜ Shan

Shi囚Zhu Li, Jing Xu, Tian『Ping W, Li囚Yong W. Yang Kun, Wang Wei, LÜ Shan.

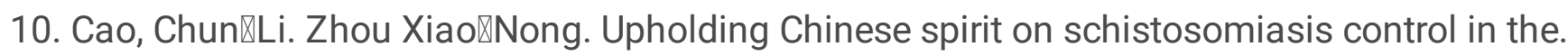

11. new era. to accel囚erate the progress towards schistosomiasis elimination in China.

12. Chin J. Schisto Control 1.1-13.

13. Zhou XN, Wang $L Y$, Chen MG, Wu XH, Jiang QW, Chen XY, Zheng J, Utzinger J. The public health significance and control of schistosomiasis-then and now. Acta Trop. 2005;96:97-105.

14. The social and economic context and determinants of Huang YX, Manderson L. 2005. The social and economic context and determinants of.

15. schistosomiasis japonica. Acta Trop. 96, 223-231. 
16. ZHOU Xiao®nong.Implementation of precision control to achieve the goal of schistosomiasis elimination in China 2016. Chin J Schisto Control, 1,1-4.

17. Ministry of Health of China. 2000. Schistosomiasis control manual[M]. Third edition.Shanghai science and technology Press. 36-39.

18. Ministry of Health of China. 2000. Schistosomiasis control manual[M]. Third edition.Shanghai science and technology Press. 52-56.

19. Katz N, Chaves A, Pellegrino J. A simple device for quantitative stool thick-smear technique in schistosomiasis mansoni.Rev. Inst Med Trop Sao Paulo. 1972;14:397-400.

20. WHO. 1985. The control of schistosomiasis: report of a WHO expert committee. WHO Tech. Rep. Ser. No. 728. World Health Organization, Geneva.

21. Celone M, Person B, Ali SM, Lyimo JH, Mohammed UA, Khamis AN, Mohammed YS, Mohammed KA, Rollinson D, Knopp S,2016. Increasing the reach: Involving local Muslim religious teachers in a behavioral intervention to eliminate urogenital schistosomiasis in Zanzibar. Acta Trop .163,142-8.

22. Bobbie P, Ali SM, A'Kadir FM, Ali JN, Mohammed UA, Mohammed KA, David R, Stefanie K. Community knowledge, perceptions, and practices associated with urogenital schistosomiasis among schoolaged children in Zanzibar, United Republic of Tanzania. PLoSNegl Trop Dis. 2016;10:e0004814.

23. Person B, Knopp S, Ali SM, A'Kadir FM, Khamis AN, Ali JN, Lymo JH, Mohammed KA, Rollinson D. 2016 Community co-designed schistosomiasis control interventions for school-aged children in Zanzibar. J Biosocial Science .48Suppl 1:S56-73.

24. Stothard JR, Khamis AN, Khamis IS, Neo CH, Wei I, Rollinson D. Health education and the control of urogenital schistosomiasis: assessing the impact of the JumanaKichocho comic-strip medical booklet in Zanzibar. J Biosoc Sci. 2016;48:40-55.

25. Parker M, Allen T, Hastings $J, 2008$. Resisting control of neglected tropical diseases: dilemmas in the mass treatment of schistosomiasis and soil-transmitted helminths in north-west Uganda. Journal of Biosocial Science.40,161 - 81.

26. Parker $M$, Allen T Does mass drug administration for the integrated treatment of neglected tropical diseases really work Assessing evidence for the control of schistosomiasis and soil-transmitted helminths in Uganda. Health research policy and systems / BioMed Central 2011.9:3.

27. Hai-ying CHEN,HU Guang-han,SONG Kuang-yu,XING Zi-wei,,HU Jia,YANG Ping-yi,PENG Guo-hau,HU, Wei-chen,,GE Jun,YU Song-shan,FU Guo-lan,LIU Zhou-hua,QI Jing-chen,WAN Bao-ping.2009. Establishment and Application of School-based Health Promotion and Intervention Model of Schistosomiasis in Lake-type Endemic Area. Chin.J Parasitol Parasit Dis.2,125-129.

28. Hu J, Zhang ZS, Hu GH, Song KY, Chen HY, Hu WC, Ge J, Peng GH, Hu ZH, Zhang J, Qiu L, Zhang J. 2009. Observation on effect of health promotion intervention for adult female residents in heavy endemic areas with schistosomiasis in Poyang Lake for 17 years.Chin. J. Zoon. 10.982-985.

29. SONG Kuang-yu YIN, Hua HU, Guang-han,CHEN Hai-ying,GE Jun,PENG, Guo-hua ZHANG, Ju,HUZhuhua,HU Wei-chen. 2010. Longitudinal observation on effect of health education for adultmale 
residents for 18 years in heavy epidemic areas of schistosomiasis around Poyang Lake region. Chin. J. Schisto. Contr. 3, 242-246.

30. Mott KE,1992. Schistosomiasis control: everybody's business. International Symposium of Schistosomiasis. Beijing, Chin. 13-29.

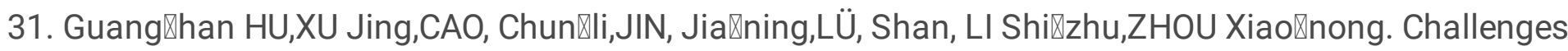
and strategies of health education and health promotion in stage of schistosomiasis elimination in China. Chin J Schisto Contr. 2018;2:117-20,123.

32. Hu GH, Hu ,J, Song KY, Lin DD, Zhang J, Cao CL, Xu J, Li D, Jiang WS. The role of health education and health promotion in the control of schistosomiasis: experiences from a 12-year intervention study in the Poyang Lake area. Acta Trop. 2005;96:232-41.

33. Guo JG, Cao CL, Hu GH, Lin H, Li D, Zhu R, Xu J. The role of passive chemotherapy plus health education for schistosomiasis control in China during maintenance and consolidation phase. Acta Trop. 2005;96:177-83.

34. Chun-li CAO, Li-juan ZHANG, BAO Zi-ping. Endemic situation of schistosomiasis in Prople's Republic of China from 2010 to 2017, Chin. J Schisto Contr. 2019;5:519-54.

35. Ti-yuan HUANG,HU Guang-han,XIE Xing-fu,XIONG Yong,CHEN Ying-ping.2012.

36. Current status of. schistosomiasis control knowledge and effect of interventions among primary school pupils in Any County,Chin. J. Schisto. Contr. 5, 609-610.

37. Guang-han HU,LIN Dan-dan,ZHANG Shao-ji,JIANG Wei-sheng, LU, Shang-biao,ZENG-Xiao-jun,KANG Zeng-hua,ZHANG Jing,LIN Han,QIU Ling.2000. Study on intervention mode of health education for schistosomiasis control. Chin. J. Schisto. Contr. 2, 65-71.

\section{Figures}




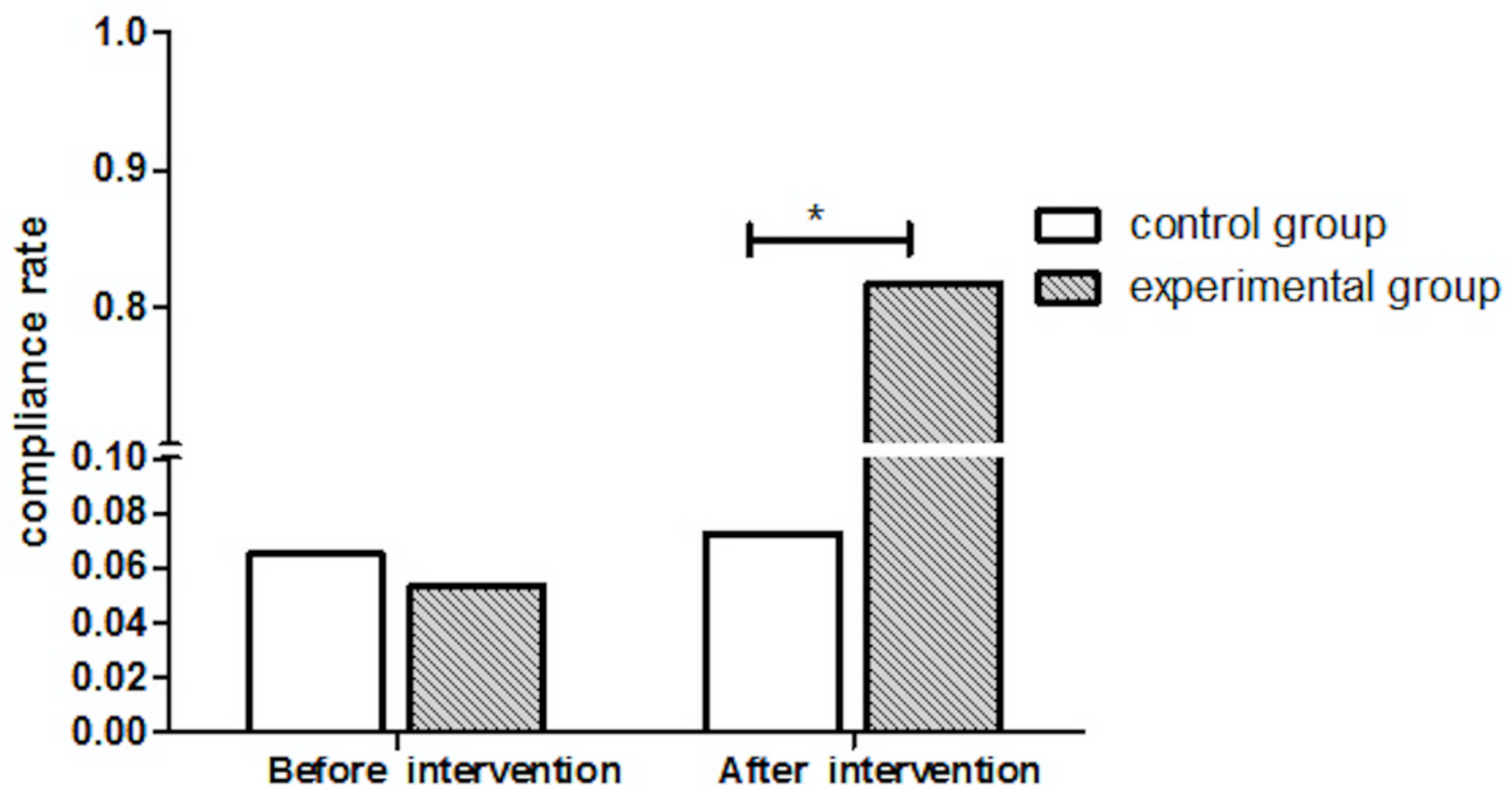

Figure 1

Compliance towards snall survey in adult villagers. 